Expert Systems in Law

Bologna, Italy - May 3-5, 1989

\title{
Design Requirements of a CD-ROM Based Reference System Capable of Replacing Print Materials in a Field of Law
}

\author{
Peter W. Martin \\ Edward Cornell Professor of Law \\ Cornell Law School, Cornell University
}

\section{l. Introduction}

Much of the research and writing on computer-based legal information systems pays little heed to the characteristics, structure, and performance of existing print-based systems. The implication, no doubt often unintended, is that print is a separate and largely irrelevant universe. ${ }^{1}$ Problems are discussed as though they sprang fresh from the effort to move information to electronic media, costs (such as the cost of adding editorial matter or indexing to stored legal documents) are dismissed as prohibitive even as print publishers continue to incur them on a routine basis, and the work patterns and format expectations of today's professionals, which are based on print materials, receive very little attention.

This paper, based on a project being carried out with support from the National Center for Automated Information Retrieval, Mead Data Central, and an IBM equipment grant through Cornell University, focuses on a pair of questions that deal with the relationship of print and computer-based materials:

(1) What elements of functionality (measured against available print materials) must a computerbased legal information system offer in order to be competitive with print?

(2) What are the more important challenges raised by moving from print to this new medium?

\footnotetext{
${ }^{1}$ There are important exceptions. See, e.g., Bing, Jon (1987), "Designing Text Retrieval Systems for 'Conceptual Searching,"' Proceedings of the First International Conference on Artificial Intelligence and Law, 43-51.
} 
The paper is written from the perspective of a pragmatic legal domain expert. I have chosen that perspective both because it fits my own experience and perspective and because it is a point of view too often overlooked in the literature on expert systems.

The approach, here, is unabashedly applied. Excellent basic research on text retrieval systems and legal expert systems has been and continues to be done - work that illuminates fundamental questions of approach or technique without offering a tool of current utility to legal professionals. Without detracting from the value of such work, this paper suggests there are important lessons to be learned from what established print-based information systems enable the lawyer or judge to do. For a computer-based system to be competitive it must do as much; it must offer as effective a means of communicating expertise, from the perspectives of both domain expert and user. Any gains a computer-based legal information system, expert system or other, purports to offer will be measured against these established information resources.

Let me begin by sketching in my perspective as domain expect. Ten years ago, having acquired substantial expertise in the field of U.S. Social Security law, I contemplated sharing that knowledge with interested professionals in the most effective way. The target group included judges, public officials, lawyers and others with a strong interest in the field. My aims included assisting decision-making and legal analysis in this field, planning for individuals potentially eligible for benefits, and advocacy on behalf of individuals who sought benefits (mapping out both the range of argument space and furnishing the materials for argument). Ten years ago, the appropriate technology for the activity I describe was the technology that legal scholars have used for centuries - book technology. I secured a tentative agreement with a law publisher for the publication of a treatise on the law of Social Security - a book that would have been rich with references to the statute, regulations, and published judicial opinions of the field. The agreement contemplated not only the initial publication of such a book, but its subsequent periodic revision. An administrative assignment interrupted the project for some eight years. When the opportunity to resume came, it seemed clear to me that there were now more effective technologies for expert communication.

For reasons on which I shall not dwell now, creation of an expert system with the capacities of those others have built using present tools was not the compelling alternative to a book to which I refer. I experimented with such systems and found them too constraining a form 
of communication for the domain expert and of uncertain attractiveness to users who are well served by print. My interest as domain expert led me to a more flexible set of communication tools. My approach was pragmatic in two related ways. First, I resisted laying down flat principles of technique of the sort many expert systems workers espouse, principles such as "no manual interventions". (A curious term, one must note, for the intellectual activity of authors, editors, and indexers.) Second, I kept steady attention focused on the print technology that was the alternative for both me and the potential users of my computer-based information system.

Since my experience and examples both derive from a single project that is not yet complete there is an inescapable tentativeness in the following observations and conclusions. For the same reason, I feel obliged to preface the discussion of the more general points to follow with a short description of this project. With assistance from the sources already noted, I have undertaken preparation of a full legal reference tool or library covering U.S. Social Security Law. This electronic library will include: a detailed statute, a more detailed set of regulations formally issued by the federal agency and, for the most part, binding on official deciders, less authoritative but nonetheless important agency materials, several thousand decisions on Social Security claims by the Federal courts at several levels, decisions extending back over the fifty year history of this national program, and finally a treatise - author prepared text that explicates the law in this area with ultimate reliance upon and frequent reference to the previously enumerated authoritative law materials. In the United States, there are print collections that attempt similar complete coverage of specialty law fields. In the field of Social Security there are at least two. The two on-line legal databases, LEXIS and WESTLAW, contain specialist law collections, but neither, as yet, has one on Social Security law. Of the listed library components, the on-line collections have only the statute, regulations, and court decisions.

With a project of this magnitude, data acquisition is a critical threshold issue. It is an issue so fundamental it receives no further discussion in this paper. Had electronic versions of the components of this projected library other than the treatise not been available to me, the venture would not have been possible. The arrangement with Mead Data Central, proprietors of the LEXIS service, under which I am proceeding, gives me a publisher or distributor of the final work, which is reassuring, and a data source which is essential. There is a second order data issue that I mention, before moving on, because it is less obvious. For some time to come, the 
benchmark version of statutes, regulations, and judicial opinions will be in print. For an electronic substitute to be competitive it must not only contain the same documents, but its versions must conform to the print editions with a very high level of accuracy. The error level in many of today's on-line legal information systems is unacceptably high.

\section{Replacing Print-Based Materials}

\subsection{The Technology Predicate}

Several developments in computer technology make it possible to contemplate creation of computer-based legal information systems that might supplant rather than merely supplement print-based materials in some areas of law. These developments include:

- The improvement of display technology to the point that it is finally reasonable to think of reading from a computer screen as being competitive with reading from a printed page, even over extended periods of time.

- Printer technology that has progressed so that the printing of selected material on demand can be fast, quiet, affordable, and of very high quality.

- Optical systems of data storage (currently CD-ROM) that have brought immense capacity to the individual workstation, again at affordable cost. This capacity is so great that virtually all print-based information in some fields of law can be stored on a single CD-ROM disk (or at most a small handful). Since 1979, the West Publishing Company has issued a set of reports containing all bankruptcy decisions of the U.S. Federal courts. The full collection now fills several bookcases. I have a colleague who ended his subscription simply because the reports were consuming so much office space. This series of decisions has just been issued in an alternative form - two CD-ROM disks with a treatise on an accompanying third disk.

Another key feature of my technology predicate is the existence and widespread acceptance of operating environments that permit manipulation of information and movement around an application to be accomplished by such intuitive and straightforward means that they become comparable to page turning and print index use. These same environments furnish simple techniques for transporting information across applications - so that a passage from 
statute, regulation, or judicial opinion can be blocked and copied into a set of notes or, ultimately, into a memorandum or brief or opinion an attorney or judge is writing.

A goal these several developments place within reach is creation of a computer-based legal information system offering access to the complete set of legal materials on a significant cluster of legal questions. In a field of law sufficiently coherent and autonomous it is now possible to contemplate building an electronic reference system that contains a full professional library for dealing with most questions the lawyer, public official, or judge must face, as well as clear direction into on-line and print materials on issues falling outside its scope. That is the opportunity which this one domain expert finds so compelling.

\subsection{The Principal Components and Functional Elements of the Typical Print-Based System in the U.S.}

To be a realistic alternative to print, such a computer- based system must incorporate the full text of all available legal documents within its scope - statutes, regulations adopted by administrative agencies, judicial and administrative opinions, and other less formal agency guidance. In addition, it must offer the range of functions provided in print by editorial additions to the legal documents (headnotes, annotations) and indexing, as well as by treatises, citators, and other forms of "secondary" legal literature. To illustrate how extensive those editorial additions are in the standard print legal reference works in the United States and how they furnish the user multiple entry points, consider three exhibits - a report of a Federal appeals court decision in a Social Security case, the annotations published in between sections of the Social Security Act, and pages from a typical treatise - again in the field of Social Security law. [Illustrations provided.] In pulling together and cross-referencing all legal materials relevant to a particular field, a computer-based system of this sort will most closely resemble the print systems, commonly called "services," published in the United States by such private firms as

Commerce Clearing House, Prentice Hall, and the Bureau of National Affairs. It must allow the user to move from an expert's exposition of the law (comparable to that furnished by a treatise, specialty service, law review article or essay accompanying an annotated law report) to the supporting or described statute, regulation, and principal decisions. Conversely, it must allow the user to begin with a legal document (statute, regulation, or decision) and move to other relevant material in ways made possible in print by an annotated statute or headnote or index. 
Finally, it must provide a means of checking to see whether subsequent legal events (such as a legislative enactment or judicial decision) have altered the effect that a particular legal document had when it first took effect. The electronic reference work that can perform all these functions offers clear gains over print alternatives. It need not reduce the need for human intellectual addition to be a superior means of communicating expertise, but the form of the medium will, perforce, alter the nature of the intellectual addition.

An electronic information system with such capacity can also embody elements of expert system, and if it does they will not appear as separate or discontinuous tools.

\section{Selected Challenges Posed by the New Medium}

\subsection{Setting Boundaries for the System}

A reference work that aims at complete coverage of a particular law domain lays high stakes on the boundary definition, for that boundary, potentially, represents a major work discontinuity for the user. This poses a challenge at both the conceptual and implementation level. Clarity as to what topics, statutory provisions, and judicial decisions lie within the domain is critically important to author and user. But even with sharply delineated scope, creating a full system using previous print and electronic sources entails major investment in finding and including all legal materials lying within it. The author, editor, or publisher of a printed book (whether treatise or set of law reports) need not address the issue of boundaries (what the work will include and what it will not) with such rigor. Concededly, any author or publisher will seek a close congruence between the scope of a work and the anticipated needs of users or readers, but the creator of a printed volume destined for a professional library can and will always take comfort in the other volumes that will reside on the same shelf. Indeed, print authors rely on other materials and cross reference to them. Despite those references, the author of a print treatise need never address the question: Have I identified all the legal source material that someone using this book will need? It is simply not the author's undertaking, nor the readers' expectation, that a treatise identify every case in point. Nearly all law books are designed to be used together with other law books. They do not need to and do not attempt to stand alone.

A vertical computer-based reference work of the sort envisioned here, however, gains

much of its advantage by holding out promise of completeness (or near completeness) and 
autonomy. That demands dramatically heightened attention to audience and function. For whom, exactly, is the work is designed? What legal questions must it cover, in what settings? In some fields of law, practice conventions and existing print materials already reflect a clear definition of scope. In others, particularly those having no print service attempting comprehensive coverage even by way of case listing or indexing, this must be developed. Few fields of law have print sources that implement a definition of scope with the thoroughness and rigor required for selection and maintenance of a full CD-ROM reference.

Let me illustrate a few different types of boundary issues using examples from the Social Security field:

The U.S. Social Security program rests on an earmarked tax imposed on wages, on employers' wage payments, and on the income of self-employed individuals. In some respects the statutory provisions that govern this tax are totally congruent with those governing benefits. Should a full Social Security reference, therefore, include coverage of the tax? (Some of the print collections in the field do, other don't.) In answering scope questions of this type, a clear vision of how the final reference work will be used and by whom is the first point of reference, a vision that derives from an understanding of the professional and institutional framework into which the reference is likely to fit. Since different government agencies and different attorneys concern themselves with tax and benefit cases in the U.S., leaving the tax materials out of a benefit-focused collection is a relatively easy conclusion. Institutional factors may lead to inclusion of materials that are, theoretically, quite separate from the core collection. For example, I have judged it important to include materials on the general statute providing for recovery of attorneys' fees in actions against the Federal government, the Equal Access to Justice Act, because recovery of fee awards in Social Security cases is so important to the segment of the profession undertaking such representation.

Other decisions about collection boundaries will in the end rest on cost-benefit determinations. A clear example of material excluded on that ground is furnished by those points, such as marital status, at which the Social Security Act incorporates state law by reference. The few cases in which any one state's domestic relations law will be determinative of Social Security entitlement laid against the massive effort that would be required to include full materials on the relevant law of fifty states dictates exclusion. 


\subsection{Achieving the Proper Balance Between Selectivity and Completeness}

All things equal, most U.S. lawyers would choose a reference system offering complete coverage of a field over one with, say, only 60 percent of the decided cases even if that 60 percent represents an expert's list of the most "important" decisions. Completeness is the great allure of the on-line systems, LEXIS and Westlaw. At the same time that lawyers crave completeness, they rely on companion systems (treatises, journal articles, annotations and other secondary sources) to identify the more important cases or statutes. In designing a complete reference system for a domain, the challenge is to superimpose selectivity on completeness. The devices that are easiest to implement are those that simply reflect the judicial structure or explicit characterization of a decision - separating decisions of a higher court from those of a lower, separating decisions that the court has indicated as being of limited application (such as the "unpublished" decisions of the U.S. Court of Appeals, 6th Circuit) from the rest. But print materials commonly offer more, namely, identification of those judicial opinions that offer an especially useful entry point to a field or set of issues or that are landmarks in the more substantive sense that they resolve doubt, reverse prior law, or lay down a new approach to be followed in the future.

In the United States, complete coverage of any field of federal law raises an issue about "unpublished" court decisions of two types. Only a fraction of federal district court opinions on legal questions are published in the principal print sources, the Federal Supplement and Federal Rules Decisions reports of the West Publishing Company. Effectively, the decision on whether to make an opinion available in those reports is made by the judge. Some judges submit a high percentage of their opinions; others, very few or none. Most specialist services in federal law fields (drawing on attorneys for the parties as the principal information source) include summaries or, in some cases, even full text access to significant numbers of "unpublished" district court decisions. The two on-line databases, LEXIS and Westlaw, include substantial numbers of such decisions. In a given field, the universe of unpublished decisions accessible through the important print services, through LEXIS, and through Westlaw will be significantly different, each one from the others.

In 1985, there were over 18,000 District Court dispositions of Social Security claims appeals. For the same year, I have identified only 256 Social Security decisions printed in the 
volumes of the West Publishing Company generally relied on by the legal profession, 129 decisions not printed by West Publishing Company but included in the LEXIS database, and over 125 decisions available in neither of those places but printed in full text or summary in the Social Security service published by another publisher, Commerce Clearinghouse. (In all, the decisions receiving public distribution in any form represent less than 3\% of all District Court Social Security decisions.) Under such circumstances, what standard of completeness should a new "full" legal reference adopt? The answer is not self-evident. On the other hand, so long as print remains the benchmark a reasonable pragmatic answer is "at least as complete as print resources."

Federal appellate decisions pose a related but different problem. With the U.S. Courts of Appeals, which hear Social Security claims on appeal from the District Courts, the historic norm has been distribution of all full appellate judgments. But in the past ten or more years, a growing fraction of the courts' appellate judgments have been designated "unpublished" and held back from the major print publisher, reflecting the courts' view that these decisions lack importance or value as precedent. Currently over fifty percent of the decisions of U.S. Courts of Appeals are "unpublished" (the figure was 61\% for 1987) but the concept and its implementation are in chaos because of electronic media. Unpublished decisions (meaning those not printed by the West publishing company) are printed in various specialty reports and loaded into computer databases. In all circuits but the 10th, roughly ninety percent of the courts' unpublished decisions are available on LEXIS. Courts need to address the issue of the precedential weight of their decisions as one distinct from publication. Until the courts rendering decisions that they consider to have no significant precedential value find an appropriate way to deal with this new form of dissemination, anyone preparing a complete library of on a Federal law topic must decide whether or not to include this large category of decisions.

\subsection{Dealing with Time (Or More Exactly the Modifying Effects of Later Legal Events on Prior Ones)}

Printed legal materials display their age. They are dated and promptly distributed. Years later, readers must form sound judgments about their continued effectiveness. The consequences of subsequent legal events are reflected in certain special tools - citators and supplements - and are reported in new editions of treatises or new codifications. In most fields, the literature of law 
is cumulative; rarely does law change in a way that allows a library or user to dispose of "obsolete" primary materials. That is not to say that obsolescence is not a real phenomenon, but rather that it occurs in so many different ways, shades, or degrees that simplistic treatment in a legal reference or full-text database is hazardous. Consider the following examples of "obsolete" primary legal materials:

- The "obsolete" judicial opinions that focus on a statutory provision that has since been altered or eliminated. Can they be safely disregarded? Sometimes those decisions represent an approach that may readily apply, by analogy, to another section of the statute.

- The "obsolete" lower court decisions that reflect approaches or a views of the law that are, in varying degrees, inconsistent with subsequent decisions in different cases by some higher court.

- Opinions that use obsolete terminology. Often there is a period when a new issue is being litigated during which courts grapple for appropriate and consistent terminology. Ultimately, they settle a shorthand phrase (the "Harris test" or the "treating physician rule") not to be found in many prior decisions reflecting variations on or specific applications of the same rule.

- Transition phenomena beyond the period of transition. A full collection of legal materials in any field based on a statute that is amended from time to time will inevitably include numerous decisions dealing primarily with problems of transition from one rule to another. Such decisions address the important question, what matters are governed by the old provision, what matters by the new; but they have passing importance. Even so, they may have enduring value on the generic questions of transition.

Most print treatises deal with these "obsolescence" or temporal problems by describing the current law, with occasional historical preambles or digressions. The latter will generally be referenced to the "obsolete" statutory provisions and decisions in illustrative, not complete, fashion. Print indices index contemporaneously and then let the user make judgments about datedness using other tools. On-line collections leave the user in much the same situation. The challenge for a complete reference using this new medium is to provide a complete collection of 
materials, old and new, with effective guidance on the current value and topical relevance of the old.

To illustrate both the problem and some of the possibilities, let me describe my Social Security decisions set - frozen as of last December. This set of decisions, complete according to a reasonable set of criteria, includes 33 decisions of the United States Supreme Court, 2,436 decisions of the United States Courts of Appeals (omitting all designated by the deciding court as lacking precedential weight), 3,898 District Court decisions published in print, and 1,011 District Court decisions not published in print but available in the LEXIS on-line system. From this total collection, I drew a core sample, all Courts of Appeals decisions of the year 1978. They number 44. If all District Court decisions were available and included in this decision set, each of these 44 appellate decisions would represent a subsequent stage of one of them, reversing, modifying, or affirming the lower court's decision. But, in fact, only five reported District Court decisions are represented by these 44 - two that were affirmed and three that were reversed.

On appeal one of the 44 decisions was, ultimately, upset by the Supreme Court, a second was explicitly disapproved by the Supreme Court in another case, and a third has been disagreed with by nearly all other circuit courts that have addressed the same issue. From this brief case review one might conclude that the sample of 44 decisions, now 10 years old, shows a very low rate of obsolescence. Of 44, 41 or 42 still stand as apparently sound precedent and only three district court decisions were, as a result of these opinions, rendered obsolete. That latter conclusion is patently unsound, since so small a percentage (under 1\%) of the District Court Social Security decisions are appealed. But more important for our present discussion are the other sources of obsolescence that have cut into the value of those ten year old decisions. In this group of Social Security decisions, like any representative sample, well over $80 \%$ of the cases involve the determination of disability. In 1979, the Social Security Administration issued a new, comprehensive set of regulations on making disability determinations. They were subsequently upheld against statutory and constitutional challenge by the United States Supreme Court. In 1984 the Social Security act, itself, was amended in ways affecting resolution of disability claims based on pain or mental impairment. Since over thirty of the 44 appeals court decisions from 1978 involve disability, some of them cases of pain or mental impairment, their value has been substantially diminished by these subsequent changes in regulation and statute. 
Any reference system, print or electronic, that does not distinguish between these decisions and ones occurring after the relevant changes for the user or note the changes and their impact falls seriously short. Some of these 1978 decisions are consistent with the regulation and statute changes, but even so, since they do not invoke present sections or terminology, they require a degree of interpretation that later decisions do not.

\subsection{Format and Structure}

An electronic collection of legal materials, stored at the workstation on CD-ROM or other similar dense medium, can offer cross-referencing possibilities only crudely suggested by such print devices as footnotes and cross-references with which lawyers are familiar, but in numbers and at speeds that can radically alter the research process. Alongside, it can offer the capacity for database searches of the type currently available on-line. The artful challenge is to design a structure that takes advantage of the enormously greater reference capability of this medium - its hypertext capability - without breaking with established professional research patterns or citation conventions. For acceptance and effective use by the present generation of legal professionals, print metaphors must be used in handling both input and output. In fields, without sophisticated print systems, the absence of such metaphors allows greater freedom, but also creates far greater problems for those who would assemble "useful" and "used" collections of material:

Before CD-ROM and hypertext can truly fit, a few database design problems have to be resolved.... [What are needed are] "conventions that work." These conventions should support the hypertext paradigm of augmenting human thinking, while including some of the familiar information-organization techniques found in existing print media... Building structure into CD-ROM hypertext databases is very important. It's analogous to the production value added to printed works by editors, designers, and publishers....CD-ROM has already won a position as an archival system for existing data. To go further, opening new markets and competing with other media, databases and retrieval systems must be specifically crafted for CDROM and the new audiences it can reach. ${ }^{2}$

${ }^{2}$ Oren, Tim (1988), "The CD-ROM Connection," Byte, Dec. 1988, 315-320. 
During a period of transition print may furnish more than metaphor; in some countries, including the United States, it imposes legal constraint. How does one refer to a decision, unit of a decision, statute or unit of a statute? Such references are necessary within the system and of course there they can be totally unintelligible to the human eye (machine but not brain executable). The issue is how one identifies a law point to another lawyer, judge, citizen - a person who may have access to the same electronic system, a different one, or to print materials. Our current citation conventions in the U.S. are premised totally on print. The most recent edition - edition 14 - of the influential Uniform System of Citation manual contains only one example of a permissible citation to an electronic legal database - in extremis, if you will. (I find delicious irony in the fact that the blue book has itself, for the au courant lawyer, been replaced by computer software designed and sold by a company that has just been acquired by LEXIS.) Needed but not yet created are conventions that are indifferent to the medium of storage and access. Each court decision should carry a unique identifier, ideally one that would provide the date and court and link to all other actions involving the same litigation. This identifier should be applied by the court to remove any issue of its being proprietary. Units of decisions should, like so much other law writing, be designated by paragraph numbers. That would permit more precise "jump" citation and eliminate the need for or need for dispute over diverse paginations a division that becomes utterly arbitrary, even meaningless, with decisions that are not distributed widely in print. The legal problem in the United States is that private print publishers of court decisions have asserted copyright protection in page divisions. So long as those page divisions remain the customary form of unit reference a very high barrier confronts those preparing electronic libraries, on-line or CD-ROM. I have referred to decisions but drafters and codifiers of statutes face a similar challenge and need for new discipline. Computer-based systems capable of following statutory cross-references require a consistency and completeness in identification of the unit being referenced - that most statutes, today, do not meet - relying as they do on print format to set off distinct provisions.

\subsection{User Interaction}

Taking advantage of a state of the art "point and click" or icon-based user interface, a CD-ROM legal reference can and should be as intuitive as page-turning and index using. It should also take advantage of user familiarity with full-text on-line legal information systems 
search techniques while reducing the need for knowing and entering command sets. Capture and transfer of information from the reference to user notes and writing should be similarly intuitive, drawing on the best of current applications in other fields for models.

\subsection{Beyond Print Substitution}

Moving to and around a full universe of important texts is the critical baseline for any computer-based reference work that would compete with print. But the computer offers more. An exciting creative challenge lies in harnessing the database management, computational, and graphic manipulation and display capabilities to provide functions that print cannot. By definition, it is here that a computer-based information system can most clearly demonstrate superiority over print materials. With a sophisticated user interface, much of this capability can be presented to the user in print-like form. The system may perform calculations specified by law on figures entered by the user and display both the steps and legal basis for them, but to the untutored user it can appear that the system is simply completing a form he or she has begun.

\subsection{Need for On-Line Connection}

Given law's seamlessness, no domain of law in the U.S. is so autonomous that it permits of a totally complete reference. Inevitably, trade-offs between the goal of comprehensiveness and the costs of including infrequently needed materials will lead to certain statutes, regulations, and decisions being left off. In the unusual case requiring reference to those materials, the user will have to go outside to other electronic resources or to print. Moreover, any reference that is distributed to users on CD-ROM or similar form will require provision for bringing research upto-date during the interval between distributions. For these reasons, a bridge between such a workstation-based reference and one of the on-line legal information systems seems an attractive, if not necessary, element. To the extent possible, this calls for minimizing the difference between access and presentation of information residing on local storage media and that accessed on-line.

\section{Conclusion}

Today, CD-ROM collections of legal materials are a present reality, not a speculative possibility, in the United States. But the phenomenon is in its infancy. The first materials to be distributed in this medium have, understandably, been print equivalents such as the Code of 
Federal Regulations or Federal Register, with search software added. ${ }^{3}$ Given effective software, even such materials offer functionality not available in print and may, for some, supplant the print alternative. But CD-ROM has far greater potential. It has the capacity to replace print materials, almost entirely, in some fields of law. ${ }^{4}$ But before it can do so, the challenges which authors, editors, and publishers of print materials have addressed, largely through complementary efforts, will have to be dealt with in novel yet familiar seeming ways. Print models may not always translate readily to this new medium, but without close attention to the conventions, functionality, and limitations of established print-based materials, creators of computer-based systems are not likely to gain wide use of them. And expert systems for law domains built without such complete and competitive information resources are themselves unlikely to achieve broad acceptance.

${ }^{3}$ Optext CFRIFR, distributed by VLS Inc., of Toledo, Ohio.

${ }^{4} \mathrm{~A}$ major step in this direction is represented by the CD-ROM Libraries released by the West Publishing Company in late 1988. The first three "libraries" contain collections of primary and secondary materials in the fields of bankruptcy, government contracts, and federal civil practice - all materials previously distributed by West in print. 